\title{
Model-free detection of synchrony in neuronal spike trains, with an application to primate
} somatosensory cortex

\author{
A. Roy ${ }^{a}$, P. N. Steinmetz ${ }^{a}, 1, K$. O. Johnson ${ }^{a}$, E. Niebur ${ }^{a}, 2$ \\ ${ }^{a}$ Krieger Mind/Brain Institute, Johns Hopkins University, Baltimore, MD 21218
}

\begin{abstract}
Synchronized neuronal firing has been reported in many neural systems and may play a role in the representation of sensory stimuli and the modification of sensory representations by both experience and attention. In this report we describe a bootstrap procedure for computing the statistical significance of changes in the degree of synchrony and apply it to recordings from the second somatosensory (SII) cortex of Macaques performing tactile and visual discrimination tasks. A majority $(68 \%)$ of neuron pairs in SII fire synchronously in response to a tactile stimulus. In a fraction of those pairs (17.5\%), the degree of synchrony covaries with the focus of attention.
\end{abstract}

Key words: synchrony, bootstrap, somatosensory cortex

1 Presently at the California Institute of Technology.

2 To whom correspondence should be addressed. 


\section{Introduction}

Synchronous firing of action potentials amongst multiple neurons is a phenomenon that has been observed in a wide range of neural systems. Temporal structures of similar nature have been proposed to play a functional role in representing sensory information, as possible representations of internal behavioral states, and in motor planning[7,1,3,4,2,11,10]. One of the challenges in the field is the development of statistical methods suitable for characterizing

the significance of synchronous firing. In the present report, we are concerned with the question whether the degree of synchrony observed changes significantly with the behavioral state of the animal. To this purpose, we develop a model-free analysis of the cross-correlogram[6]. Significance was tested by bootstrap procedures at two levels using appropriate null-hypotheses.

\section{Experimental Methods}

The activity of a total of 553 pairs of neurons in SII from 2 awake behaving monkeys was recorded using 7 extracellular electrodes driven individually [9]. Physiological methods were similar to those described by [8]. The experimental protocol required both monkeys to alternate between performing a tactile discrimination task (considered the "attentive" task for recordings in somatosensory cortex) and a visual ("nonattentive") task. 


\section{Statistical Methods}

\subsection{Test for significance of the degree of synchrony}

The spike trains of two neurons $\alpha$ and $\beta$ recorded simultaneously are denoted as $S_{\alpha s m}^{n}(t)$ and $S_{\beta s m}^{n}(t)$. Here, $n=1 \ldots N_{s m}$ indexes the trial number, for a particular behavioral state $m$ and stimulus $s$, and $t=1 \ldots T / b$ the bin number where $T$ is the length of the spike trains and $b$ the bin-width. The raw cross-correlogram $C_{\alpha \beta s m}^{n}(\tau)$ averaged over $N_{s m}$ trials is

$$
C_{\alpha \beta s m}(\tau)=\frac{1}{N_{s m}} \sum_{t} \sum_{n} S_{\alpha s m}^{n}(t+\tau) S_{\beta s m}^{n}(t)
$$

In order to subtract the contribution of stimulus locked mean rate effects from the raw cross-correlogram, the shift predictor (or shuffle predictor) is subtracted, yielding the covariogram $\zeta_{\alpha \beta s m}(\tau)$

$$
\zeta_{\alpha \beta s m}(\tau)=C_{\alpha \beta s m}(\tau)-\frac{1}{N_{s m}\left(N_{s m}-1\right)} \sum_{i \neq j} \sum_{t} S_{\alpha s m}^{i}(t+\tau) S_{\beta s m}^{j}(t)
$$

The stimulus-averaged covariogram $\zeta_{\alpha \beta m}(\tau)$ for a particular behavioral state $m$ is computed as the average of Eq. 2 over all $S_{m}$ stimuli in this state.

Our first objective is to test whether the covariogram for small time shifts (around $\tau=0$ ) for a specific neuron pair is significantly above noise. We define the deviation of the covariogram from zero (i.e the degree of synchrony) as the sum-squared area under the covariogram in a $50 \mathrm{msec}$ time window 
around $\tau=0$ and refer to it as to our statistic $S$. In particular, let us denote the observed statistic $S\left[\zeta_{\alpha \beta m}(\tau)\right]$ as $Z_{\alpha \beta m}$. The significance of the number of excess coincidences was tested using a bootstrap ${ }^{3}$ method [5]. We use the null hypothesis $H_{o}$ that the two neurons $\alpha$ and $\beta$ are independent. Let $\Pi_{N_{s m}}$ denote a permutation of $N_{s m}$ trials for stimulus $s$ and behavioral state $m$. We generate for each iteration $S_{m}$ such permutations and use them to compute a bootstrapped covariogram $\zeta_{\alpha \beta s m}^{\Pi_{N_{s m}}}(\tau)$ for each stimulus. The covariogram $\zeta_{\alpha \beta m}^{\Pi}(\tau)$ for a particular iteration under $H_{o}$ is computed by averaging over all $S_{m}$ such covariograms. The significance level of the observed statistic $Z_{\alpha \beta m}$ is then tested against the distribution of $\tilde{Z}_{\alpha \beta m}\left(S\left[\zeta_{\alpha \beta m}^{\Pi}(\tau)\right]\right)$ computed from all $S_{m}$ iterations to determine the probability that the data could have arisen assuming $H_{o}$ is true. No a priori assumptions are required about the underlying nature of the random process generating the spike trains, particularly the independence of firing in neighboring bins of the binned spike trains or the distribution of the test statistics, contrary to what is assumed in many statistical models.

\subsection{Significance of Attentional Effect}

Our second objective is to determine whether attention has a significant effect on synchronous firing. Only pairs of neurons which possess a significant degree of synchrony according to the test in section 3.1 were included.

$\overline{3}$ More precisely, a Fisher permutation test; results are similar with bootstrapping. 
Single trial covariograms for each stimulus and behavioral type were computed as in section 3.1. An appropriate null hypothesis for this test is that the degree of synchrony is independent of behavioral state or presented stimulus. Again, our measure of the degree of change in synchrony, our statistic $D$, was the sum squared deviations between the averaged covariograms in a 50 msec time window centered around zero time shift. In order to test the difference in synchrony as a function of one of the behavioral states (attended vs. unattended), indexed as $m=1$ or 2 , we compute the observed statistic as:

$$
Z_{\alpha \beta}=D\left[C_{\alpha \beta 1}(\tau)-C_{\alpha \beta 2}(\tau)\right]
$$

If the null hypothesis is true, covariograms obtained by averaging over trials during the tactile task should not be different, except for random variations, from those obtained in the baseline condition, the visual task. Let $\Pi_{n}$ denote a set of $n$ trials drawn with replacement from the set of visual trials and $C_{\alpha \beta}^{\Pi_{n}}(\tau)$, the averaged covariogram over this set. We use a Monte-Carlo simulation to estimate the distribution of the bootstrapped test statistic, defined as

$$
\tilde{Z}_{\alpha \beta}\left(N_{t}, N_{v}\right)=D\left[C_{\alpha \beta}^{\Pi_{N_{t}}}(\tau)-C_{\alpha \beta}^{\Pi_{N_{v}}}(\tau)\right]
$$

with $N_{t}=\sum_{s=1}^{S_{m}} N_{s 1}$ and $N_{v}=\sum_{s=1}^{S_{m}} N_{s 2}$. The significance level of $Z_{\alpha \beta}$ is then tested against the distribution of $\tilde{Z}_{\alpha \beta}\left(N_{t}, N_{v}\right)$. 
Table 1

\begin{tabular}{|c|c|c|c|}
\hline Monkey & Synchrony & Change & Increase \\
\hline \hline Monkey1 & $113 / 145(80 \%)$ & $41 / 113(36 \%)$ & $37 / 41(90 \%)$ \\
\hline Monkey2 & $264 / 408(65 \%)$ & $25 / 264(9 \%)$ & $17 / 25(68 \%)$ \\
\hline Total & $377 / 553(68 \%)$ & $66 / 377(17.5 \%)$ & $54 / 66(82 \%)$ \\
\hline
\end{tabular}

The second column indicates the fraction of cell pairs which showed significant synchrony $(p<0.05)$ for each monkey. The third column shows the fraction of those which showed a significant change in the synchrony $(p<0.05)$ with the attentional state. Finally, the fourth column indicates the percentage in which synchrony in SII increased with attention directed on the tactile task.

\section{Results}

We applied the above statistical procedures to spike data recorded from macaque area SII while the animals were performing attentional tasks. Table 1, Col. 2 shows the number of neuron pairs that showed a significant peak in the covariogram (all significances reported at $p<0.05$ ). Overall, $68 \%$ of neuron pairs in SII showed a significant degree of synchronous firing. Column 3 shows the number of pairs that had a significant change in the degree of synchronous firing between the attended and unattended tasks. Overall, $17 \%$ of the pairs selected in the first test show a significant change in the degree of synchrony with the amount of attention focused on the tactile stimulus. Furthermore, we found that of all pairs with a significant change, $82 \%$ had a greater degree of synchronous firing during performance of the attented tactile detection task than during the visual task (Col. 4). 


\section{Discussion}

In complex nervous systems, behavior is not only influenced by the immediate sensory input but also by the internal cognitive or perceptive state of the animal. It has been suggested on theoretical grounds that the temporal structure of spike trains plays a role in the coding of such states and that the correlational structure of spike trains varies with the attentional state of the animal[10]. We test this prediction by analysing spike trains recorded from the cortex of an awake behaving monkey working in a task under attentional control. We show that statistically significant increases in the synchrony of neurons in somatosensory are positively correlated with the level of attention devoted to tactile stimuli. These changes in synchronization are evidence for a functional role of synchronous firing in processing sensory information.

\section{Acknowledgements}

We thank Steve Hsiao and Paul Fitzgerald for the data used in this work and acknowledge support by NSF and the Sloan Foundation.

\section{References}

[1] M. Abeles. Role of the cortical neuron: Integrator or coincidence detector? Israel J. Med. Sci., 18:83-92, 1982. 
[2] M. Abeles. Corticonics - Neural circuits of the cerebral cortex. Cambridge University Press, 1991.

[3] J.E. Dayhoff and G.L. Gerstein. Favored patterns in spike trains. I. Detection. J. Neurophysiol., 49:1334-1348, 1983.

[4] J.E. Dayhoff and G.L. Gerstein. Favored patterns in spike trains. II. Application. J. Neurophysiol., 49:1349-1363, 1983.

[5] B. Efron and R. J. Tibshirani. An Introduction to the Bootstrap. Chapman and Hall, New York, NY, 1993.

[6] J. J. Eggermont. The correlative brain : theory and experiment in neural interaction. Springer Verlag, Berlin - New York, 1990.

[7] G.L. Gerstein and W.A. Clark. Simultaneous studies of firing patterns in several neurons. Science, 143:1325-1327, 1964.

[8] S. S. Hsiao, D. M. O'Shaughnessy, and K. O. Johnson. Effects of selective attention on spatial form processing in monkey primary and secondary somatosensory cortex. J. Neurophysiology, 70(1):444-447, 1993.

[9] V.B. Mountcastle, H.J. Reitboeck, G.F. Poggio, and M.A. Steinmetz. Adaptation of the Reitboeck method of multiple microelectrode recording to the neocortex of the waking monkey. J. Neurosci. Methods, 36:77-84, 1991.

[10] E. Niebur and C. Koch. A model for the neuronal implementation of selective visual attention based on temporal correlation among neurons. Journal of Computational Neuroscience, 1(1):141-158, 1994. 
[11] E. Niebur, C. Koch, and C. Rosin. An oscillation-based model for the neural basis of attention. Vision Research, 33:2789-2802, 1993.

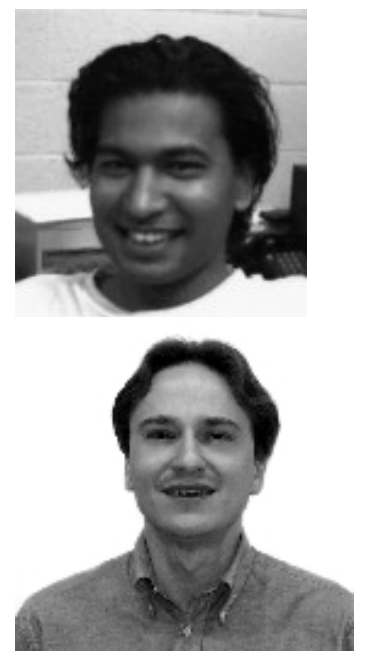

Arup Roy got a B.Tech in Instrumentation Engineering from I.I.T Kharagpur (India), and then a M.S. in Biomedical Engineering from Virginia Commonwealth University. Currently he is a Ph.D. candidate for Biomedical Engineering at the Johns Hopkins University. His interests lie in the field of computational neuroscience.

Peter N. Steinmetz is a computational neuroscientist at Caltech. He is a post-doctoral scholar in the Division of Biology and the Computation and Neural Systems Program. Dr. Steinmetz received his M.D. and Ph.D. in Biomedical Engineering from the Johns Hopkins University. He is interested in the statistics of neural coding and the biophysics of computation. His recent work involves determining how accurately pyramidal cells can compute given their intrinsic noise sources.

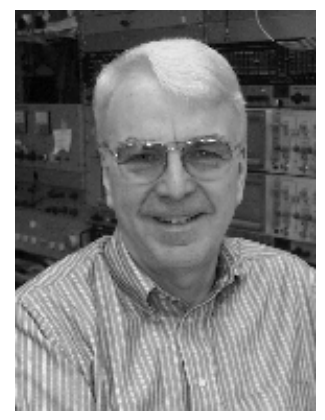

Ken Johnson got a B.S. in Electrical Engineering in 1961 and then worked in the space program for several years. In 1970 he got a Ph.D. in Biomedical Engineering from the Johns Hopkins University where his thesis research consisted of reconstruction of the neural population activity evoked by a vibratory stimulus on the skin. Since then he has worked on the neural mechanisms of tactile perception. The work has involved neurophysiological, psychophysical, and computational investigations in equal measure.

Ernst Niebur was trained as a physicist at the Universities of Dortmund (W. Germany; Diplom Physiker 1982) and Lausanne (Switzerland; Dr. ès sciences 1988). He then joined the Computation and Neural Systems group at Caltech where he was a Research Fellow and Senior Research Fellow. He is presently an Assistant Professor in the Department of Neuroscience and the Krieger Mind/Brain Institute at Johns Hopkins University. 